\section{$\underset{\substack{\text { hommes } \\ \text { \& migrations }}}{ }$}

\section{Hommes \& migrations}

Revue française de référence sur les dynamiques

migratoires

$1317-1318 \mid 2017$

L'Europe en mouvement

\title{
Le camp de Grande-Synthe : l'humanitaire aux deux visages
}

\section{Alexia Duytschaever et Chloé Tisserand}

\section{(2) OpenEdition \\ Journals}

Édition électronique

URL : http://journals.openedition.org/hommesmigrations/3898

DOI : 10.4000/hommesmigrations.3898

ISSN : 2262-3353

Éditeur

Musée national de l'histoire de l'immigration

Édition imprimée

Date de publication : 1 avril 2017

Pagination : 118-122

ISBN : 978-2-919040-38-4

ISSN : $1142-852 X$

\section{Référence électronique}

Alexia Duytschaever et Chloé Tisserand, « Le camp de Grande-Synthe : I'humanitaire aux deux visages », Hommes \& migrations [En ligne], 1317-1318 | 2017, mis en ligne le 01 avril 2017, consulté le 15 septembre 2020. URL : http://journals.openedition.org/hommesmigrations/3898 
LE CAMP DE GRANDESYNTHE : L'HUMANITAIRE AUX DEUX VISAGES 
A utomne 2015, le campement de la zone du Basroch, situé en centre-ville de GrandeSynthe, a vu sa population s'accroître considérablement en l'espace de trois mois, entre août et décembre 2015. Le nombre d'exilés est passé de 250 à environ 2 500-3 ooo personnes. La plupart sont des Kurdes venus de Syrie, d'Irak, d'Iran... On trouve des familles, des hommes seuls, des mineurs isolés. Le camp du Basroch incarne l'absence de l'État sur la question de la prise en charge de ces exilés. Les médias se sont empressés de dénommer le campement la « new jungle » en référence au bidonville calaisien et ont relayé des images de tentes et de pieds d'enfants s'enfonçant dans la boue, la neige, où l'on distingue aussi des palettes en bois et l'accumulation des déchets. En octobre 2015, les riverains signent une pétition pour dénoncer leur quotidien devenu invivable et lâchent: " On nous avait promis un écoquartier, on a un bidonville'. " Le maire de Grande-Synthe, Damien Carême, d'Europe écologie les verts et fondateur du réseau des élus hospitaliers, a pour projet la construction de 500 logements au sein d'un écoquartier sur cette zone d'activité, la première phase de 200 logements est prévue entre 2017-2019.

\section{Mise à l'abri des plus fragiles en dépit de la volonté de l'État}

Le maire écologiste s'est associé à l'ONG Médecins sans frontières (MSF) pour mettre à l'abri la population du campement à l'approche de I'hiver. Leur solution, temporaire, a pris la forme d'un "premier camp humanitaire de France ", modélisé selon les normes internationales dictées par l'ONU et qui sera installé à la Linière, une ancienne ferme excentrée située à proximité du chemin de fer et en contrebas de l'autoroute. Le camp, qui s'étend sur un kilomètre de long, était d'abord composé de rangées de tentes, dont une centaine a été soufflée en février par la tempête. Elles ont finalement été remplacées par 375 chalets en bois offrant une capacité totale d'accueil de 2500 personnes. Le coût de cette démarche représente 3,1 millions d'euros, la majorité des fonds provenant de Médecins sans frontières (2,6 millions d'euros), la ville et la communauté urbaine de Dunkerque ont complété (500 000 €). Cette initiative est allée à l'encontre de la politique générale de l'État qui se résume le plus souvent au démantèlement des campements, squats, jungles et qui n'encourage donc pas l'initiative d'une création de camp, même « humanitaire ", souhaitant plutôt déplacer les exilés vers des centres d'accueil et d'orientation (CAO), les centres d'accueil pour demandeurs d'asile (Cada) et les centres de rétention administratifs (CRA). "Je pallie une faille de l'État ${ }^{2}$ ", a déclaré le maire de Grande-Synthe, loué pour avoir tenu tête à l'État en voulant donner aux exilés un accueil digne. Pour autant, au-delà de ce décor idyllique, la gestion des migrants à Grande-Synthe (environ 21000 habitants) se révèle ambiguë.

\section{Un projet, deux visions de «l'humanitaire»}

Depuis son ouverture en mars 2016, le camp de la Linière a vécu des jours mouvementés et sa gestion a été l'enjeu central du rapport de force que se sont livrés l'État, la ville et les associations. Bien que modélisé par MSF, la gestion du camp a été à l'origine confiée à l'association bretonne Utopia 56, qui mobilise essentiellement des volontaires bénévoles et est connue pour gérer le Festival des Vieilles Charrues. Des cuisines communautaires, des espaces de vie avec prises électriques sont montés, les migrants se sont approprié les chalets en graffant des motifs sur les façades et en cultivant des jardinières. Les associations historiques se plaignent d'un ama- 


\section{SPÉCIAL FRONTIÈRES}

teurisme de la part d'Utopia $56^{3}$. De plus, en mai, la ville de Grande-Synthe n'a pas les moyens de financer son camp humanitaire (4 millions d'euros de fonctionnement) et sollicite l'État qui accepte et pose ses conditions. II est décidé de remplacer les « bénévoles » d'Utopia 56 par des gestionnaires " professionnels ", l'association AFEJl, qui mobilisent tout un ensemble de travailleurs sociaux (médiateurs, éducateurs, assistants sociaux). Ce type de mise en opposition des bénévoles versus les professionnels n'est d'ailleurs pas anecdotique. Elle rappelle les vifs débats internes au sein du champ du travail humanitaire : d'un côté, les partisans de la professionnalisation revendiquent une " efficacité et une opérationnalité " accrue comparé à "l'amateurisme " des bénévoles et, d'un autre côté, les défenseurs du volontariat mettent en avant la «déshumanisation du geste humanitaire » que peut provoquer une trop grande "opérationnalité ${ }^{4}$ ». La différence à Grande-Synthe, c'est que ce débat sort un tantinet de son champ habituel, pour mettre en opposition les bénévoles humanitaires avec cette fois-ci des professionnels du social. Ce sont bien deux visions distinctes de l'aide qui sont mises en œuvre : on a d'abord une politique du refuge (MSF/Utopia 56) qui repose sur le volontariat et une revendication "d'humanité " partagée avec les migrants (l'accès aux droits, le relogement etc.), puis on observe une politique de la régulation (ville/État) qui repose sur des « professionnels " dont le but est aussi de mettre à l'abri les migrants, mais surtout de contrôler leur présence sur le territoire en les accompagnant vers d'autres structures d'accueil (CAO, Cada, etc.).

\section{La «position médiane » du travailleur social}

Grande-Synthe pose la question de l'aide humanitaire et du rôle que celle-ci est censée jouer dans le contrôle des populations migrantes en Europe.
Une fois nommé gestionnaire, les responsabilités de l'AFEJI semblent avoir changé. Des frictions ont surgi autour du règlement intérieur du camp : en juillet, le sous-préfet a écrit une note indiquant que le camp n'est ouvert que pour les demandeurs d'asile, ce qui sera démenti par une autre note de la ville et de MSF qui reprécisent qu'il est aussi ouvert pour les migrants en partance pour l'Angleterre. Les professionnels du social paraissent se trouver dans une position d'entre-deux.

Des médiatrices ont regretté que leur mission sociale se transforme en acte de contrôle : par exemple, il leur est demandé de vérifier l'intérieur des " shelters " (abris) et ensuite, s'ils sont vides, de les condamner temporairement en vissant l'entrée. L'objectif serait d'empêcher quiconque d'investir les lieux sans autorisation et d'endiguer un phénomène discret relatif aux activités des passeurs. Les camps dans le Dunkerquois sont marqués par leur présence. En janvier 2016, on recensait trois blessés, dont un par balles, lors d'une rixe. Sur le camp de la Linière, les passeurs ont la mainmise sur les chalets qui sont monétisés. En les fermant, c'est-à-dire en assurant une mission qui ne relève pas du social, les travailleurs sociaux se retrouvent dans des situations tendues et en porte-à-faux avec la population à laquelle ils doivent être attentifs au quotidien.

La fermeture progressive des shelters s'opère également en vue de réduire les places vacantes, dans le cadre du démantèlement du camp souhaité par l'État. Depuis la création du camp jusqu'au mois de septembre, une soixantaine de chalets ont déjà été retirés. "On a basculé dans une logique plus dure où nous sommes moins bien vus auprès des exilés, indique une des médiatrices du camp. Avant on avait le temps, on s'asseyait, on buvait un thé avec eux, ce n'est plus le cas. On a l'impression que les choses nous échappent. " Alors que le camp était ouvert à tous, les professionnels doivent désormais refuser les nouveaux arrivants, des hommes seuls, interdits d'entrée, 
qui se présentent à l'accueil. En septembre, le camp est apparu triste, l'humanité tant prônée bien morne, les espaces de vie partagés sont désertés, les cuisines collectives peu utilisées. Les forces de l'ordre campent devant l'entrée, plus loin une barrière est tenue par des agents de sécurité et du personnel de l'AFEJI qui contrôlent les entrées en notant le nom de chaque visiteur extérieur. Environ cinquante associations interviennent en marge des actions du gestionnaire, sans compter les particuliers.

Cette posture, entre social et contrôle, place le professionnel dans une " position médiane », idée développée par David Gonin pour décrire dans un autre contexte la position des soignants en prison : "L'organisation médicale constituerait alors une sorte d'élément tiers entre la communauté de détenus et celle du personnel de surveillance ${ }^{5}$. " Les professionnels du social du camp de Grande-Synthe semblent se situer dans cet entre-deux délicat, entre à la fois les exilés et les exigences de l'institution qui ne vont pas toujours dans leur sens. Myriam Hachimi Alaoui et Alexandra Nacu se sont intéressées à la prise en charge sanitaire des personnes irrégulières et ont fait part des dilemmes rencontrés par les soignants, justement parce qu'il semble exister un télescopage de l'administration sur le soin. "Leur marge de manœuvre s'est affaiblie, elle se heurte aux impératifs financiers de l'institution auxquels s'ajoutent les contraintes qu'imposent les dernières politiques d'immigration ${ }^{6}$. " Les soignants sont confrontés à des situations difficiles et mobilisent d'autres recours que la logique professionnelle afin que leur patient ait un accès aux soins. II semble que l'on retrouve ce type de stratégies à Grande-Synthe : certains professionnels ont décidé de quitter l'AFEJI car ils ne se sentent plus en accord avec ces nouvelles responsabilités, qui sont susceptibles de contredire les raisons pour lesquelles ils se sont investis sur le camp de la Linière. Ils laissent entendre qu'ils n'ont pas vraiment « signé pour faire ça ». D’autres mettent en place des formes de compensation : lorsqu'ils se trouvent obligés de refuser de distribuer des couvertures à des résidents du camp, ils essayent de leur donner de la nourriture, du tabac, ce qu'ils peuvent. Un don effectué à titre individuel, pour compenser les limites d'une générosité institutionnelle.

\section{Une solution " digne» sans être vraiment « normale »}

Le camp humanitaire de Grande-Synthe semble être apparu comme le contre-modèle de ce qui a été construit par l'État à Calais. Médiatiquement, l'un semblait incarner l'humanité, l'autre la sécurité. À Calais, I'État a conçu un centre d'accueil provisoire (CAP, 18 millions d'euros) à l'aide de 125 conteneurs sécurisés de $14 \mathrm{~m}^{2}$ qui peuvent accueillir au total 1500 personnes et sont posés dans une zone contrôlée entourée de grillages. Dans les dortoirs, on trouve des lits superposés, de l'électricité et du chauffage, on y entre par identification par paume de la main et un code confidentiel. À GrandeSynthe, le maire veut que le camp soit ouvert, MSF souhaite que les populations se sentent comme chez elles. Les lieux peuvent être personnalisés, des navettes sont mises en place pour acheminer les migrants vers Auchan. La solution du " camp humanitaire ", imaginé avec MSF, ne convainc pas pour autant les initiateurs du projet : « Ce n'est ni la jungle, ni un camp, mais un accueil digne pour des réfugiés même si ce n'est pas encore une solution normale. On ne se glorifie pas de monter un camp humanitaire ", indique le directeur de cabinet du maire qui préférerait voir les exilés insérés dans le droit commun. Comme le souligne Smaïn Laacher, le clandestin l'est parce qu'il relève de l'urgence humanitaire $^{7}$ et, tant 


\section{SPÉCIAL FRONTIÈRES}

qu'il le restera, il continuera d'être l'indésirable, celui qui stagne dans un monde en marge ${ }^{8}$. Bien qu' " humanitaire ", un camp reste un camp, c'està-dire un "logement a minima ", une " solution élémentaire " immédiate et sommaire ${ }^{9}$. Mais, à Grande-Synthe, c'est le "système débrouille " de bonnes volontés individuelles qui a été mis en place localement pour répondre à l'urgence humanitaire. Le quiétisme étatique qui a duré de la construction de ce camp humanitaire jusqu'à la signature de la convention tripartite qui a fait entrer l'État dans le dispositif tend à donner raison à Éric Fassin et Aurélie Windels ${ }^{10}$ qui décrivaient en 2014 une "politique d'auto-expulsion » exercée à l'encontre des migrants roms. Une politique de l'inaction sociale, du contrôle et de la bureaucratie, qui se fonde sur l'espoir d'encourager les populations migrantes à partir volontairement du territoire, à « re »-partir dans leur pays d'origine.

\section{Les Grand-Synthois et la migration}

Le parti pris de la mairie d'agir sans l'appui de l'État n'a pas seulement eu des conséquences sur le quotidien des migrants dans sa commune, il a aussi très certainement pesé sur l'opinion publique. Des habitants rencontrés dans un bar du centre-ville semblent approuver cette action. Ils précisent, fiers mais non sans humour, que le maire est d'ailleurs en lice pour devenir le "meilleur maire du monde ». Il faut préciser que les exilés sont invisibles dans le centre-ville et, lorsqu'on évoque la situation migratoire à Calais, les habitants admettent craindre un démantèlement de la "new jungle $e^{11}$ " de Calais, car le camp de Grande-Synthe agit comme un vase commu- niquant avec la ville des Six Bourgeois. Les GrandSynthois ont peur de vivre la même situation que les Calaisiens (10 ooo migrants selon les associations, heurts entre exilés et policiers, tensions entre exilés et riverains). Toujours est-il que la façon de gérer le problème migratoire contribue à amplifier ou à amoindrir les peurs émanant de la population. Louise Tassin ${ }^{12}$, qui a travaillé sur l'aversion de la population à l'égard des migrants sur l'île de Lampedusa, montre en premier lieu que le centre qui a été créé dans les années 1990 était un centre de rétention. Sa médiatisation a terni l'image de l'île dont la ressource principale est le tourisme, ce qui a éveillé une hostilité des habitants à l'égard des migrants. Cette animosité s'est calmée en 2007 lors de la création d'un nouveau centre, un centre de premier secours et d'accueil (CPSA) où les habitants de Lampedusa sont apparus comme un emblème de l'accueil. Ce centre crée des emplois et embauche des habitants de Lampedusa pour entretenir le site. En 2012, le maire écologiste de Lampedusa prône un meilleur accueil des migrants et l'institution du centre semble vouloir inculquer l'ethos de l'accueil aux habitants de Lampedusa en leur demandant d'avoir une conduite et des propos irréprochables à l'égard des migrants, une sorte de "paternalisme social ". Mais Louise Tassin montre aussi les failles de ce dispositif : I'aversion demeure auprès des habitants de Lampedusa et se lit à travers notamment le dégoût des misérables et la haine de l'indocile. À Grande-Synthe, le climat, bien qu'apaisé entre habitants de la ville et résidents du camp, apparaît tout de même comme un statu quo fragile entre deux populations qui finalement se croisent sans vraiment se connaître.

8. Michel Agier, Le couloir des exilés. Être étranger dans un monde commun, Bellecombe-en-Bauges, éd. du Croquant, 2011. 9. Marc Bernardot, "Campement d'infortunes, figures, topiques, politiques", in Fiona Meadows (dir.), Habiter le campement, Paris, Cité de l'Architecture/Acte Sud, 2016. 10. Éric Fassin, Aurélie Windels, Roms et riverains. Une politique municipale de la race, Paris, La Fabrique éditions, 2014. 11. La "new jungle » de Calais a été démantelée après cette étude en octobre 2016. 12. Louise Tassin, "Accueillir les indésirables. Les habitants de Lampedusa à l'épreuve de l'enfermement des étrangers ", in Genèses, vol. 3, n 96, 2014, pp. 110-131. 